\title{
Radiofrecuencia pulsada del ganglio de la raíz dorsal en el síndrome radicular lumbosacro crónico refractario a los esteroides epidurales
}

\author{
P. Castromán ${ }^{1}$, F. Cristiani², M. Surbano², S. Ayala², A. Schwartzmann² y G. Varaldi ${ }^{3}$ \\ ${ }^{1}$ Profesor Agregado, ${ }^{2}$ Profesor Adjunto y ${ }^{3}$ Asistente. Departamento y Cátedra de Anestesiología. Hospital \\ de Clínicas. Facultad de Medicina. Universidad de la República, Montevideo, Uruguay
}

\section{ABSTRACT}

Introduction: Approximately $20 \%$ of patients with chronic lumbosacral radicular syndrome do not respond to epidural steroid injections. Pulsed radiofrequency of the dorsal root ganglion (DRG) is proposed as an alternative treatment.

Objetive: To evaluate the analgesic effect of pulsed radiofrequency of the DRG in patients with chronic lumbosacral radicular syndrome, refractory to epidural steroid injections.

Patients and method: 31 patients with chronic lumbosacral radicular syndrome that failed to at least two epidural steroid injections, received pulsed radiofrequency of the DRG, utilizing two cycles of 120 seconds, 45 $\mathrm{V}$ and $42{ }^{\circ} \mathrm{C}$. Evaluation was carried out a month later. Brief Pain Inventory was applied before procedure and one month later. A reduction of 2 points in the verbal numerical scale (VNS) assessed with the question 6 of the Brief Pain Inventory, was considered a satisfactory analgesic response. Intensity and Interference Scores were calculated.

Results: The VNS score decreased from $7.7 \pm 2,2$ to $5.9 \pm 3$ (p<0.01). Intensity and Interference Scores were reduced from $7.1 \pm 1.5$ to $5.9 \pm 2.1(p<0.01)$ and $7.1 \pm 1.9$ to $6.0 \pm 2.3$ ( $p<0.02$ ) respectively. In 12 patients $(40 \%$ ) a satisfactory analgesic response was observed. In these patients the VNS decreased from $8.0 \pm 1.5$ to $3.8 \pm 2.6$. Intensity and Interference Scores were reduced from $7.4 \pm 1.3$ to $3.9 \pm 1.5$ ( $p<0.0001$ ) y $7.7 \pm 1.9$ to $4.9 \pm 2,7$ ( $p=0.002$ ) respectively.

Conclusions: DRG pulsed radiofrequency produce a mild but statistically significant reduction in VNS and pain interference, in patients with chronic lumbosacral radicular syndrome. However, in $40 \%$ of the patients

Castromán P, Cristiani F, Surbano M, Ayala S, Schwartzmann A y Varaldi G. Radiofrecuencia pulsada del ganglio de la raíz dorsal en el síndrome radicular lumbosacro crónico refractorio a los esteroides epidurales. Rev Soc Esp Dolor 2019;26(3):166-174.

\section{RESUMEN}

Introducción: Aproximadamente, el $20 \%$ de los pacientes con síndrome radicular lumbosacro crónico no responden a las inyecciones epidurales de esteroides. La radiofrecuencia pulsada del ganglio de la raíz dorsal (GRD) es una alternativa terapéutica en ellos.

Objetivos: Evaluar el efecto analgésico de la radiofrecuencia pulsada del GRD en pacientes con síndrome radicular lumbosacro crónico, refractario a los esteroides epidurales.

Material y método: 31 pacientes con síndrome radicular lumbosacro crónico que no respondieron a dos inyecciones epidurales de esteroides fueron tratados con radiofrecuencia pulsada del GRD a $45 \mathrm{~V}$ y $42{ }^{\circ} \mathrm{C}$, en 2 ciclos de 120 segundos. Se utilizó el Inventario Abreviado de Dolor para evaluar los resultados. Se consideró una respuesta satisfactoria al procedimiento una disminución de dos puntos en la escala verbal numérica (EVN) evaluada en la pregunta 6 del inventario abreviado de dolor. Los índices de intensidad y de interferencia fueron calculados antes y después del tratamiento.

Resultados: La EVN se redujo de 7,7 \pm 2,2 a 5,9 \pm 3 ( $p<0,01)$. Los índices de intensidad e interferencia disminuyeron de $7,1 \pm 1,5$ a $5,9 \pm 2,1(p<0,01)$ y $7,1 \pm 1,9$ a $6,0 \pm 2,3(p<0,02)$ respectivamente. 12 de los 30 pacientes (40\%) presentaron una respuesta analgésica satisfactoria. En estos, la EVN disminuyó de $8,0 \pm 1,5$ a $3,8 \pm 2,6$ y los índices de intensidad $e$ interferencia de 7,4 $\pm 1,3$ a 3,9 $\pm 1,5(p<0,0001)$ y $7,7 \pm 1,9$ a 4,9 $9 \pm 2,7$ ( $p=0,002)$, respectivamente.

Conclusiones: La aplicación de radiofrecuencia pulsada en el GRD reduce en forma discreta pero estadísticamente significativa la intensidad del dolor y su interferencia funcional en pacientes con síndrome radicular lumbosacro refractario a las inyecciones epidurales de
Recibido: 07-08-2018

Aceptado: 27-11-2018

Correspondencia: Pablo Castroman pablocastro227@gmail.com 
a satisfactory analgesic effect was observed. In some of them this changes persist after 3 months of the procedures.

Key words: Lumbosacral radicular pain, pulsed radiofrecuency, dorsal root ganglion.

\section{INTRODUCCIÓN}

El síndrome radicular lumbosacro se caracteriza por dolor lumbar irradiado a uno o más dermatomas lumbares o sacros, comúnmente denominado lumbociatalgia. La base fisiopatológica de este tipo de dolor es la irritación por inflamación o compresión de las raíces nerviosas comprometidas (1). Las causas del mismo son variadas, siendo las hernias de disco y las protrusiones discales más frecuentes en los pacientes menores de 50 años y los cambios degenerativos en la columna lumbar, como por ejemplo la estenosis foraminal, en los pacientes mayores de esa edad (1-3). Cuando el tratamiento no invasivo basado en distintos regímenes farmacológicos y la fisioterapia no logra alcanzar alivio satisfactorio del dolor o adecuada recuperación funcional, las técnicas intervencionistas deben considerarse $[3,4]$. Dentro de ellas, la inyección epidural de esteroides es una de las técnicas indicadas más frecuentemente, basada en un supuesto proceso inflamatorio presente en el conflicto disco-radicular (3-5). Sin embargo, aproximadamente un $20 \%$ de los pacientes con síndrome radicular lumbosacro presenta respuestas poco satisfactorias a este tratamiento (6). Los motivos de estos resultados pueden ser diversos, incluyendo el carácter crónico del dolor radicular con escasa participación de la inflamación, con un papel predominante de los fenómenos neuropáticos. En estos casos, la cirugía de columna se ofrece con frecuencia como propuesta terapéutica (6). Sin embargo, esta opción está muchas veces limitada por la edad y/o el estado físico del paciente, o incluso por las propias preferencias de este sobre los tratamientos ofrecidos.

Una alternativa a la opción quirúrgica para el tratamiento del síndrome radicular lumbosacro, cuando la misma no está indicada o se prefiere evitar, es la aplicación de radiofrecuencia pulsada sobre los ganglios de la raíz dorsal (GRD) de las raíces involucradas (6). Existen varios reportes sobre la utilización de este tratamiento aplicado al dolor radicular crónico, tanto cervical como lumbar, con escasa respuesta a otras modalidades de tratamiento (6-10). Según la evidencia disponible, el alivio del dolor y la mejora en la repercusión funcional del mismo oscilan en un 50-60 \%, no reportándose hasta el momento complicaciones con el uso de la técnica en esta modalidad (6-10). En nuestro medio, la experiencia con este procedimiento es escasa y no ha sido reportada. esteroides, al mes de realizada. En aproximadamente el $40 \%$ se obtuvieron respuestas analgésicas satisfactorias que, en algunos casos, se extendió a tres meses tras realizar el procedimiento.

Palabras clave: Síndrome radicular lumbosacro, radiofrecuencia pulsada, ganglio de la raíz dorsal.
El objetivo de nuestro estudio es la evaluación del efecto analgésico de la radiofrecuencia pulsada del GRD en pacientes con síndrome radicular lumbosacro crónico refractario a las inyecciones epidurales de esteroides, utilizando el Inventario Abreviado de Dolor (Brief Pain Inventory] como herramienta de evaluación de resultados.

El perfil de efectos colaterales y complicaciones de la técnica fueron también consignados.

\section{MATERIAL Y MÉTODO}

Se trata de un estudio prospectivo, cuasi-experimental, para evaluar el efecto de la radiofrecuencia pulsada de los GRD a nivel lumbar en una población seleccionada con síndrome radicular lumbosacro crónico unilateral refractario a las inyecciones epidurales de esteroides, a causa de hernia discal, protrusiones discales lumbares o estenosis del canal, en los cuales la cirugía de columna fue previamente descartada.

El Inventario Abreviado de Dolor se utilizó como instrumento para evaluar el dolor de los pacientes seleccionados. Este cuestionario de autollenado permite a los pacientes cuantificar la intensidad de su dolor y el grado en el cual este interfiere con aspectos emocionales y funcionales. Además, el cuestionario consta de ítems adicionales que evalúan el nivel subjetivo de alivio que el tratamiento proporciona, la localización del dolor y la descripción del mismo. El cuestionario se basa en preguntas vinculadas a la intensidad del dolor y de cómo este afecta a la vida de la persona en distintos aspectos, graduándose del 0 al 10. El análisis de ambas dimensiones permite obtener los índices de intensidad y de interferencia. El índice de intensidad surge de promediar las respuestas vinculadas a la intensidad del dolor, mientras que el de interferencia de promediar las respuestas vinculadas con las repercusiones funcionales y afectivas que dicho dolor produce (11-13).

La pregunta número 6 del cuestionario se refiere a la intensidad del dolor en el momento en el que se aplica el cuestionario. Puede equivalerse a la aplicación de la escala verbal numérica (EVN), utilizada con frecuencia como instrumento de evaluación de resultados. El cuestionario se aplicó previamente a la realización del procedimiento, considerándose como situación basal, y se repitió en el control al mes de realizado el mismo. En aquellos pacientes en los cuales fue consignado un cambio de, al menos, dos puntos en la EVN en el primer control, se realizaron nuevos controles a los tres meses 
de ejecutado el procedimiento. Aquellos pacientes que en el primer control no presentaron cambio alguno en el EVN, se les repitió un nuevo procedimiento de radiofrecuencia pulsada. Los efectos de este segundo procedimiento no fueron incluidos en este trabajo.

Los criterios de inclusión fueron síndrome radicular lumbosacro de seis o más meses de evolución, con una resonancia magnética nuclear con evidencia de hernia de disco, protrusión discal o estenosis del canal, que hubieran recibido al menos dos inyecciones epidurales de esteroides con una respuesta no satisfactoria. En todos los casos, se realizaron previamente inyecciones epidurales de esteroides, por abordaje interlaminar parasagital en primera instancia, seguido de una segunda inyección por vía transforaminal al mes de la primera. Estas inyecciones fueron realizadas utilizando asistencia radiológica. Se estableció como respuesta no satisfactoria a los esteroides epidurales una disminución menor a dos puntos en la EVN (pregunta 6 del cuestionario] al mes de realizado el último procedimiento de inyección [14]. Después del fracaso de estas, la opción quirúrgica fue descartada por los neurocirujanos o traumatólogos de columna del hospital.

Se tomaron los siguientes criterios de exclusión: pacientes menores de 18 años y mayores de 80, embarazadas, lumbalgia sin irradiación radicular, dolor radicular lumbar bilateral, dolor de menos de seis meses de evolución, cáncer, aplastamientos vertebrales, diabetes tipo I, presencia de marcapasos o cardio-desfibriladores implantados.

El estudio fue aprobado por el Comité de Ética del Hospital de Clínicas. Todos los pacientes firmaron el formulario de consentimiento informado habitualmente utilizado para el procedimiento a realizar.

El procedimiento se realizó en el quirófano, en posición decúbito ventral. Se colocó vía venosa periférica y monitorización estándar. Se utilizó el arco en C para la localización de los GRD mediante los enfoques anteroposterior, oblicuo y perfil (Figuras 1 y 2). Se utilizó un generador de radiofrecuencia de la marca Cosman, modelo G4. Se utilizaron cánulas de radiofrecuencia número 22 o 20, de 10 o $15 \mathrm{~cm}$ de longitud respectivamente, según la contextura física del paciente, con $1 \mathrm{~cm}$ de punta activa. La cánula de radiofrecuencia fue colocada en la cara antero-superior o techo de los neuroforámenes seleccionados (Figura 1). En el caso de S1 se optó por colocar la cánula en el primer agujero sacro y realizar la radiofrecuencia de la raíz nerviosa, en lugar de realizar el abordaje del ganglio de la raíz dorsal a través del hueso sacro, por considerarse este excesivamente invasivo. Se profundizó la aguja hasta una línea imaginaria situada en la mitad del espesor del hueso sacro (Figura 2).

Se identificó el GRD correspondiente mediante la estimulación sensitiva, debiendo ser positiva entre 0,3 y 0,6 mV y la respuesta motora a la estimulación, negativa a voltajes no menores al doble de los utilizados para obtener la respuesta sensitiva. Se realizó radiofrecuencia pulsada durante 120 segundos, a $45 \mathrm{~V}$ y 42 grados de temperatura, después de la inyección de $1 \mathrm{ml}$ de suero fisiológico para la reducción de impedancia. Terminada esta, se repitió un nuevo pulso con las mismas características que el anterior. El procedimiento se realizó en los niveles previamente seleccionados. Los pacientes se controlaron al mes del procedimiento en la consulta externa de nuestro hospital. Las variables utilizadas para medir la efectividad de la intervención fueron: escala verbal numérica del 0 al 10 (EVN,

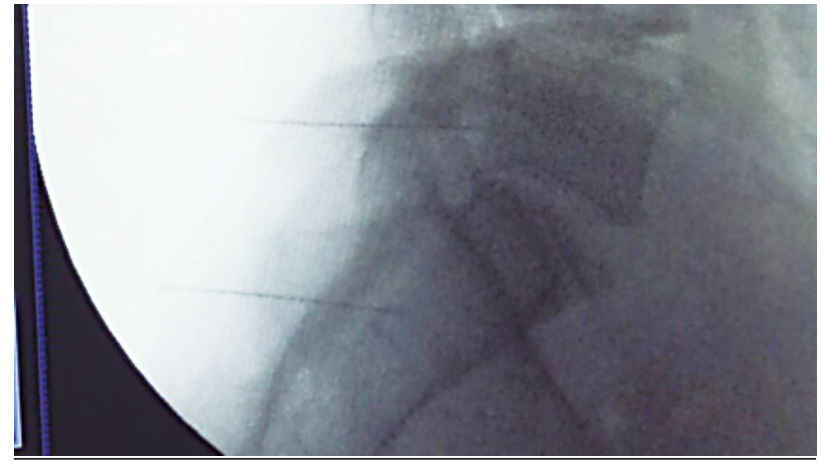

Fig. 1. Vista radiológica de perfil que muestra una cánula del ángulo anterosuperior o techo del neuroforamen de L5 y S1, topografía aproximada del ganglio de la raíz dorsal. En S1 se observa la cánula colocada a través del primer agujero sacro hasta aproximadamente una línea imaginaria que pasa por la mitad del espesor del hueso sacro con la finalidad de realizar la radiofrecuencia pulsada a la raíz nerviosa.

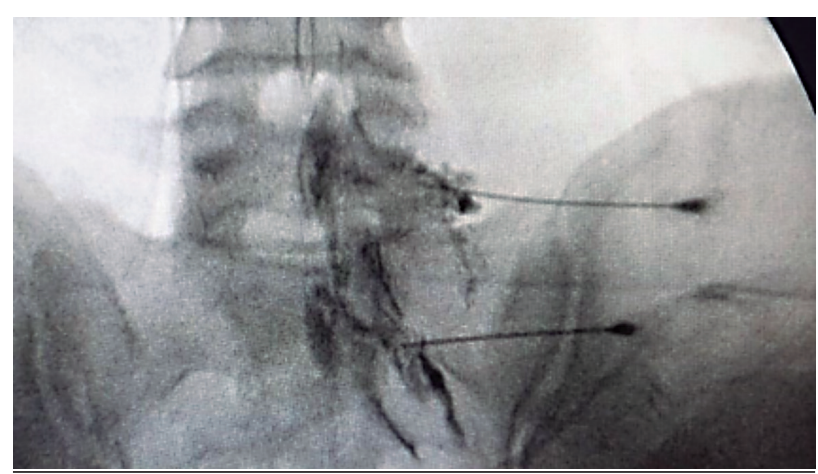

Fig. 2. Imagen radiológica anteroposterior de una cánula de radiofrecuencia posicionada en neuroformen L5-S1 y S1 después de inyección de contraste.

pregunta 6 del cuestionario). Se definió como respuesta positiva una disminución de 2 puntos en esta escala. Se determinó el número de pacientes con respuesta positiva y la variación del promedio de los puntajes en la EVN, antes y después del procedimiento. Se promediaron los índices de intensidad e interferencia antes y después del procedimiento. Finalmente se consignó la percepción de mejoría con el tratamiento, proporcionado por el inventario abreviado de dolor (IAD), expresado por el paciente en porcentaje [O \% no mejoró, $100 \%$ mejoría completa). Aquellos pacientes con respuestas positivas fueron nuevamente evaluados con el IAD a los tres meses de realizar el procedimiento. Los pacientes que no respondieron al procedimiento se les ofreció la repetición del mismo, siendo excluidos de posteriores evaluaciones en este estudio. Los datos se expresaron como la media y su desvío estándar. Para evaluar estadísticamente las diferencias entre los datos antes y después del tratamiento, se utilizó el test de t para muestras dependientes. Las gráficas y el análisis 
estadístico fue realizado con el programa Graphpad Prism Version 7.0. Se consideró estadísticamente significativo en valor de p menor de 0,05.

\section{RESULTADOS}

Entre los meses de diciembre del 2015 y diciembre de 2017 se incluyeron 46 pacientes que fueron atendidos en el Servicio de Tratamiento del Dolor Crónico con síndrome radicular lumbosacro, que habían sido tratados con inyecciones epidurales de esteroides con respuesta no satisfactoria. De estos, 31 completaron la valoración al mes, 12 presentaron respuestas satisfactorias y, de estos, 8 pacientes completaron la valoración a los tres meses. Los cuatro pacientes restantes con respuestas satisfactorias no retornaron al segundo control. Un caso fue descartado por presentar en la evaluación posterior dolor facetario predominante.

De los 30 pacientes evaluados (23 mujeres y 7 hombres], en 18 pacientes se realizó radiofrecuencia pulsada de L4 y L5, en 10 solo de L5, y en 2 de L5 y S1. La totalidad de los pacientes presentaban protrusiones discales en la resonancia magnética nuclear, con grados variables de compromiso del canal lumbar y en un caso había cirugía previa de columna. No hubo ningún caso de hernias discales. El promedio de tiempo de evolución del dolor fue de 42 meses.

El puntaje en la EVN previo al procedimiento se redujo de $7,7 \pm 2,2$ a $5,9 \pm 3$ ( $p<0,01$, test de $t$ para muestras pareadas], tomando en cuenta la totalidad de la muestra ( $n=30$ ), lo que implica una variación de $23 \%$ [Tabla I, Figura 3]. El índice de intensidad disminuyó de 7,1 $\pm 1,5$ a 5,9 $\pm 2,1(p<0,01)$, lo que implica un cambio del $16 \%$, mientras que el índice de interferencia descendió un $15 \%[7,1 \pm 1,9$ a 6,0 $\pm 2,3]$ ( $p<0,02$ ) (Tabla I, Figuras 4 y 5). Al evaluar los pacientes que respondieron favorablemente utilizando el EVN, 12 de 30 pacientes mostraron un descenso de, al menos, 2 puntos en dicha escala, lo que representa un $40 \%$ de la muestra. Si analizamos a estos pacientes por separado, podemos observar que los valores iniciales de EVN disminuyeron de 8,0 $\pm 1,5$ a 3,8 $\pm 2,6$ después del procedimiento, lo que implica un porcentaje de reducción de 53 \% (Tabla II, Figura 6).

TABLA I

ESCALA VERBAL NUMÉRICA (EVN), ÍNDICES DE INTENSIDAD E INTERFERENCIA BASALES Y AL MES DEL TRATAMIENTO EN EL TOTAL DE LOS PACIENTES $(N=30)$. SE EXPRESA COMO LA MEDIA Y DESVÍO ESTÁNDAR

\begin{tabular}{|c|c|c|}
\hline Pain Inventory & Basal & 1 mes \\
\hline EVN & $7,7 \pm 2,2$ & $5,9 \pm 3$ \\
\hline Índice de intensidad & $7,1 \pm 1,5$ & $5,9 \pm 2,1$ \\
\hline Índice de interferencia & $7,1 \pm 1,9$ & $6,0 \pm 2,3$ \\
\hline
\end{tabular}

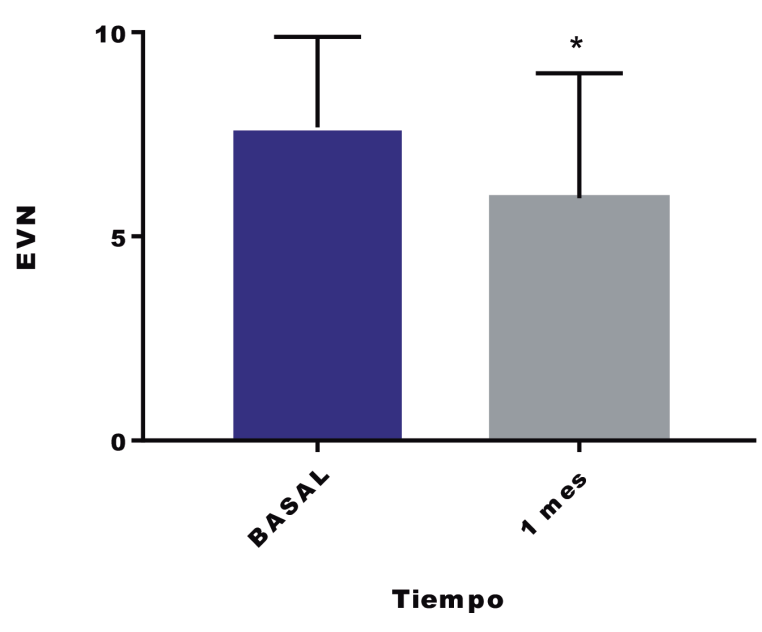

Fig. 3. Valores en la escala verbal numérica (EVN) obtenidos de la pregunta 6 del inventario abreviado de dolor (IAD) basales y al mes de la realización de radiofrecuencia pulsada del GRD en la totalidad de la muestra $(n=30)$. Se observa un descenso de un $23 \%$ al mes con respecto a los valores basales, que es estadísticamente significativo $(p<0,01$, test de $T$ para muestras pareadas].

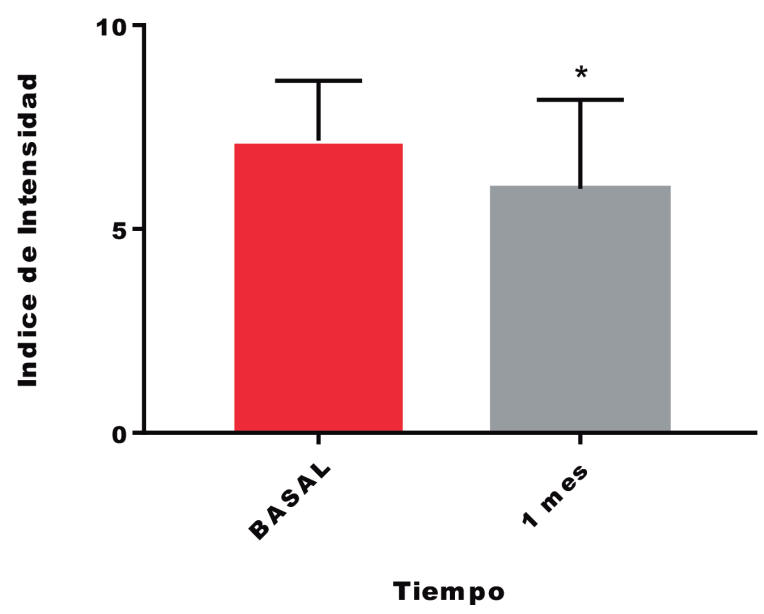

Fig. 4. Valores del índice de intensidad obtenidos del inventario abreviado de dolor (IAD) basales y al mes de la realización de radiofrecuencia pulsada del GRD en la totalidad de la muestra $(n=30)$. Se observa un descenso de un $16 \%$ al mes con respecto a los valores basales, que es estadísticamente significativo $(p<0,01$, test de T para muestras pareadas]. 
De estos 12 pacientes, 10 tuvieron una variación de tres puntos o más en la EVN (más de $50 \%$ de variación), mientras que solo dos tuvieron una variación de dos puntos en la escala (30 \% de variación). En este grupo de pacientes los índices de intensidad y de interferencia se redujeron un 47 y $36 \%$, respectivamente, pasando de 7,4 $\pm 1,3$ a 3,9 $\pm 1,5(p<0,0001)$ para el índice de intensidad y de 7,7 $\pm 1,9$ a 4,9 $\pm 2,7$ ( $p=0,002$ ) para el índice de interferencia (Tabla II, Figuras 7 y 8). Los 30 pacientes incluidos refirieron un promedio de mejoría subjetiva de $59 \%$. En ningún caso se registraron complicaciones de los procedimientos.

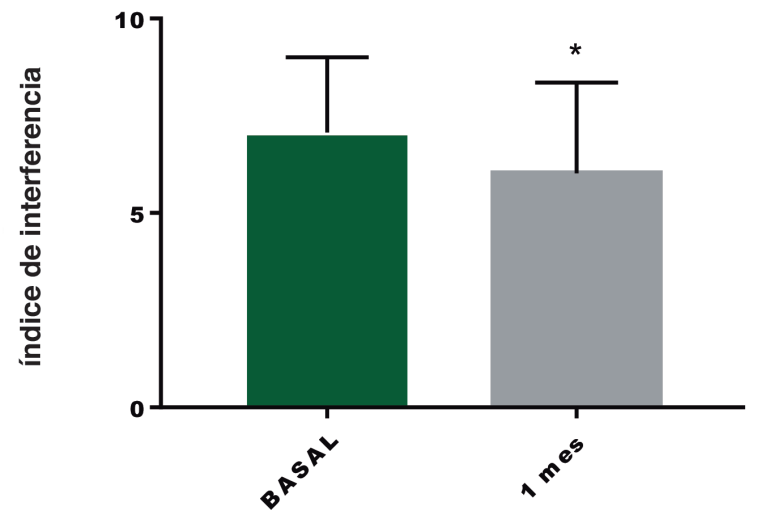

Tiempo

Fig. 5. Valores del índice de interferencia obtenidos del inventario abreviado de dolor (IAD) basales y al mes de la realización de radiofrecuencia pulsada del GRD en la totalidad de la muestra ( $n=30$ ). Se observa un descenso de un $15 \%$ al mes con respecto a los valores basales, que es estadísticamente significativo ( $p<0,01$, test de T para muestras pareadas).

TABLA II

ESCALA VERBAL NUMÉRICA, ÍNDICES DE INTENSIDAD E INTERFERENCIA BASALES Y AL MES DEL TRATAMIENTO EN PACIENTES CON RESPUESTA SATISFACTORIA A LA RFP ( $N=12$ ). SE INCLUYE LA VALORACIÓN A LOS TRES MESES EN 8 PACIENTES. SE EXPRESA COMO LA MEDIA Y DESVIIO ESTÁNDAR

\begin{tabular}{|c|c|c|c|}
\hline $\begin{array}{c}\text { Pain } \\
\text { Inventory }\end{array}$ & Basal & 1 mes & $\begin{array}{c}3 \text { meses } \\
(n=8)\end{array}$ \\
\hline EVN & $8,0 \pm 1,5$ & $3,8 \pm 2,6$ & $3,8 \pm 3,3$ \\
\hline $\begin{array}{c}\text { Indice de } \\
\text { Intensidad }\end{array}$ & $7,4 \pm 1,3$ & $3,9 \pm 1,5$ & $4,3 \pm 1,6$ \\
\hline $\begin{array}{c}\text { Indice de } \\
\text { Interferencia }\end{array}$ & $7,7 \pm 1,9$ & $4,9 \pm 2,7$ & $5,4 \pm 2,5$ \\
\hline
\end{tabular}

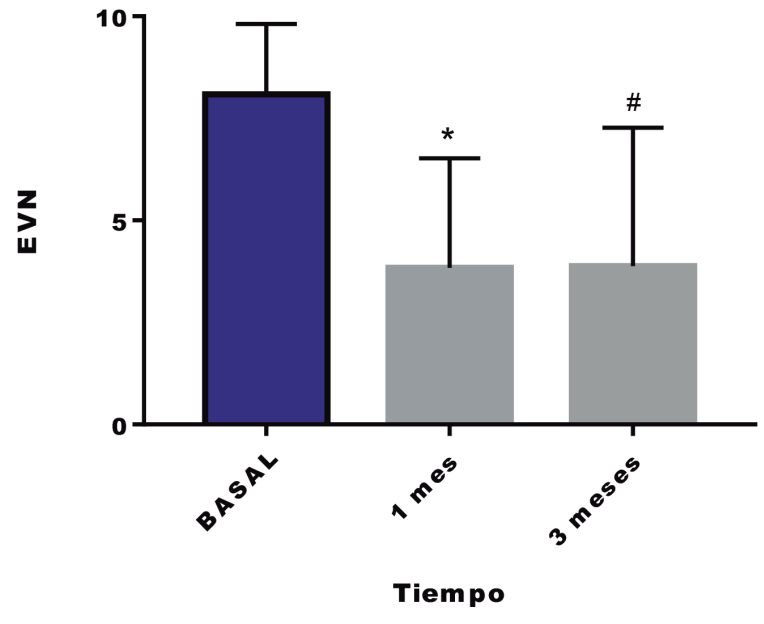

Fig. 6. Valores en la escala verbal numérica obtenidos del inventario abreviado de dolor (IAD) basales y al mes de la realización de radiofrecuencia pulsada del GRD en aquellos pacientes con respuesta satisfactoria como se describe en el texto [ $n=12,40 \%$ ). Se observa un descenso de un $53 \%$ al mes con respecto a los valores basales. En ocho de estos pacientes se controlaron también a los tres meses, manteniéndose la mejoría observada al mes. Para ambos controles las diferencias fueron estadísticamente significativas $(p<0,01$, test de $T$ para muestras pareadas].

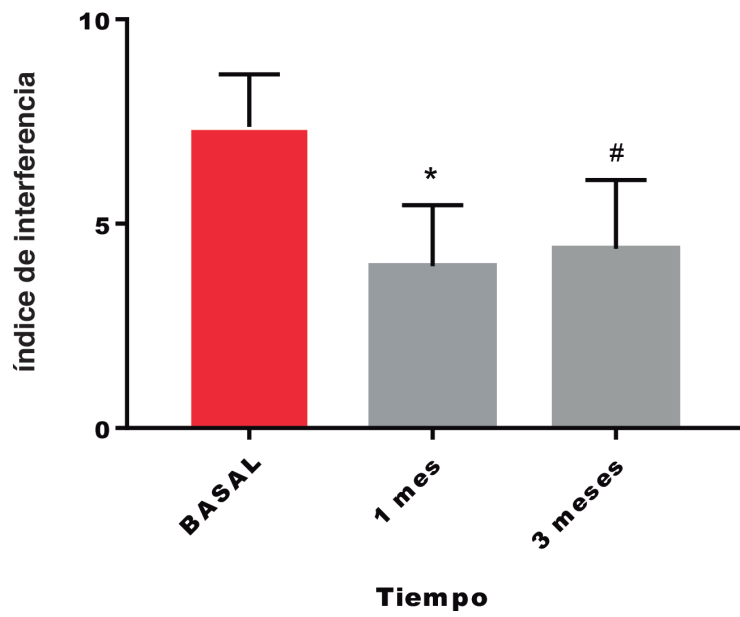

Fig. 7. Valores en el índice de intensidad obtenidos del inventario abreviado de dolor (IAD) basales y al mes de la realización de radiofrecuencia pulsada del GRD en aquellos pacientes con respuesta satisfactoria como se describe en el texto ( $n=12,40 \%$ ). Se observa un descenso de un $53 \%$ al mes con respecto a los valores basales. En 8 de estos pacientes se controlaron también a los tres meses, manteniéndose la mejoría observada al mes. Para ambos controles las diferencias fueron estadísticamente significativas ( $p<0,01$, test de T para muestras pareadas). 


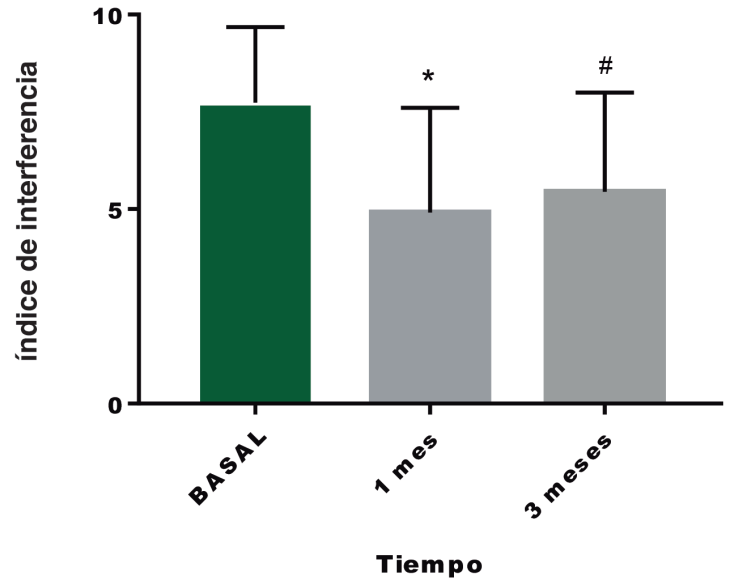

Fig. 8. Valores en el índice de interferencia obtenidos del inventario abreviado de dolor (IAD) basales y al mes de la realización de radiofrecuencia pulsada del GRD en aquellos pacientes con respuesta satisfactoria como se describe en el texto ( $n=12,40 \%)$. Se observa un descenso de un 36 $\%$ al mes con respecto a los valores basales. En ocho de estos pacientes se controlaron también a los tres meses, manteniéndose la mejoría observada al mes. Para ambos controles las diferencias fueron estadísticamente significativas ( $p<0,01$ y $p<0,05$, test de T para muestras pareadas).

En ocho pacientes con respuesta satisfactoria al mes fue posible una evaluación a los tres meses de los procedimientos. En estos, la EVN se mantenía en valores bajos, $3,8 \pm 3,3$ ( $p=0,007$ ) ocurriendo algo similar con los índices de intensidad e interferencia, siendo de 4,3 $\pm 1,6$ y 5,4 $\pm 2,5$, respectivamente ( $p=0,001$ y $p=0,02$, cuando se lo compara con los valores basales, Figuras 6, 7 y 8).

El grupo de pacientes con respuestas analgésicas satisfactorias no presentó diferencias significativas con el grupo de pacientes con escasa respuesta al tratamiento en factores como la edad, género, tiempo de aparición del dolor o niveles lumbares tratados.

\section{DISCUSIÓN}

Tomando el grupo de pacientes en su totalidad, la aplicación de un procedimiento de radiofrecuencia pulsada produce una reducción estadísticamente significativa, aunque clínicamente moderada, de la intensidad y de la interferencia del dolor en las actividades diarias de los pacientes, al mes de realizado el tratamiento. Tomando como referencia el EVN en este grupo, se produjo un descenso de 1,8 puntos después del tratamiento. Farrar y cols. examinaron 10 estudios clínicos con un total de 2724 pacientes con polineuropatía diabética, neuralgia postherpética, lumbalgia, fibromialgia y osteoartritis, utilizando al EVN antes y después de un determinado tratamiento y una escala de 7 puntos en la que se valoraba la impresión global del cambio y que iba desde "mucho mejor" a "mucho peor". Las reducciones del dolor en 2 puntos o en un $30 \%$ se asociaban a "bastante mejor" (15).

Numerosos estudios han examinado la magnitud de los cambios en el inventario abreviado de dolor asociados a distintos tratamientos, y los resultados han demostrado mejorías de 1 a 3 puntos, dependiendo del tratamiento y de la patología de base. La información disponible sugiere que un cambio de 1 punto del score de interferencia sería un objetivo razonable para estudios diseñados para identificar los cambios mínimos clínicamente importantes (13). En nuestro grupo de pacientes, el índice de interferencia se redujo 1,1 puntos.

Si se considera exclusivamente aquellos pacientes con respuestas satisfactorias como fueran definidos previamente (disminución de 2 puntos en la EVN), los resultados de este estudio muestran una reducción satisfactoria en el $40 \%$ de los pacientes (12 de 30 ), quienes pasaron de presentar un dolor severo EVN $=8,0$ ) a un dolor leve a moderado (EVN $=3,8$ ). Este grupo de pacientes se benefició además de una reducción de $36 \%$ en el índice de interferencia, lo que se traduce en una mejoría en la calidad de vida de los pacientes al reducir el grado en el que el dolor interfiere con las actividades diarias de los mismos. De los 12 pacientes que mejoraron en nuestra muestra, 10 presentaron una mejoría de $50 \%$ o más, y los dos restantes de un $30 \%$. En 8 de estos pacientes la mejoría se extendió a los tres meses del primer procedimiento.

Consideramos estas respuestas como aceptables, teniendo en cuenta que para procedimientos intervencionistas sobre la columna vertebral se considera una respuesta mínima satisfactoria, un cambio de por lo menos un 30 \% con respecto a la valoración inicial después de la realización de los mismos $(14,15)$. También destacamos que la mejoría de un $50 \%$ o más en la mayor parte de los pacientes que respondieron aleja el efecto placebo de los resultados, efecto que no puede descartarse en modelos cuasi-experimentales, como el del presente estudio.

Si comparamos nuestros resultados con los de otros trabajos, el porcentaje de éxito observado es más bajo. En 65 pacientes con síndrome radicular lumbar en los que se realizó radiofrecuencia pulsada en el GRD, Van Boxem encuentra una respuesta analgésica positiva en el $55,4 \%$ de los pacientes. Los autores consideran una respuesta positiva a una reducción de 2 puntos en una escala del 0 al 10, después de seis semanas del procedimiento (10).

En otro estudio realizado por estos mismos autores, un $29 \%$ de los pacientes que recibieron radiofrecuencia pulsada como parte del tratamiento de su dolor radicular, mejoraron sus puntuaciones en una escala de 0 a 100, aunque con una exigencia de un $50 \%$ de cambio como criterio de respuesta satisfactoria [7].

En otro estudio, publicado por Trinidad y cols., 26 pacientes con dolor radicular en lista de espera para operarse de columna recibieron radiofrecuencia pulsada del GRD. Al evaluar los pacientes al año, 19 pacientes no requirieron de cirugía, debido a la mejoría obtenida con el procedimiento intervencionista. 
En estos pacientes se observó una disminución promedio de 2,95 puntos en la escala numérica. Destacamos que en este trabajo se utilizaron 6 minutos de radiofrecuencia pulsada (16).

La principal diferencia entre el presente estudio y los trabajos consultados es que en estos no se realizaron previamente inyecciones epidurales de esteroides como parte de tratamiento. En nuestro caso, se incluyeron pacientes en los cuales habían fallado tanto las estrategias farmacológicas como las intervencionistas de uso corriente (inyecciones epidurales de esteroides por vía interlaminar y transforaminal] y en los cuales la cirugía de columna no fue indicada por distintas razones, quedando entonces pocas opciones disponibles para el tratamiento del dolor.

Es difícil establecer los motivos por el cual un grupo de pacientes en nuestra muestra respondió favorablemente al tratamiento y otro grupo no (un total de 18 pacientes). Nuestro estudio no permite establecer una asociación entre variables como tiempo de duración del dolor o diagnóstico radiológico, con las respuestas favorables. Si bien no se encontraron trabajos en la bibliografía que mostraran dicha asociación, se ha descrito una relación entre bajos puntajes en la escala de valoración del pensamiento catastrófico sobre el dolor (Catastrophizing Pain Scale] y altos puntajes en escalas de diagnóstico de dolor neuropático como el DN4 y la escala de LANSS (Leeds Assessment of Neurophatic Symptoms and Signs] con respuestas exitosas después de la aplicación de radiofrecuencia pulsada en los GRD $(10,17)$. Van Boxem, por su parte, encuentra en un estudio reciente que la edad mayor a 55 años, un bajo grado de afectación funcional y una respuesta positiva a un bloqueo diagnóstico previo se asocian con un porcentaje mayor de respuestas positivas a la realización de radiofrecuencia pulsada del GRD en pacientes con síndrome radicular lumbosacro (18). Sin embargo, no encontró que altos puntajes en la escala de DN4 se relacionaran con mejores respuestas a la radiofrecuencia pulsada del GRD. Lee y cols., por su parte, encuentran una diferencia costo-beneficio negativo de los bloqueos diagnósticos realizados previo a la radiofrecuencia pulsada del GRD, por lo cual no los recomiendan (19). En un estudio retrospectivo, Kim y cols. encuentran que la presencia de dolor osteomuscular asociado y una buena respuesta a las inyecciones epidurales de esteroides pueden considerarse factores predictores de respuestas satisfactorias a la radiofrecuencia pulsada del GRD [20].

Parece claro que son necesarios nuevos estudios, con un número mayor de casos, para determinar aspectos vinculados con la selección de pacientes candidatos al procedimiento para lograr porcentajes mayores de éxito.

No existieron complicaciones en los pacientes tratados, y si bien la muestra es pequeña, se puede inferir que se trata de una técnica segura, ya que en ninguno de los trabajos consultados se describen complicaciones $(6-10,16,21)$.

Existe controversia acerca del tiempo de duración de la radiofrecuencia y su relación con el resultado del procedimiento. En el presente estudio se utilizó 4 minutos, pero existen otros autores que proponen sesiones más prolongadas (16). Se han descrito también protocolos en los cuales se asocia a la radiofrecuencia pulsada la inyección de una mezcla de esteroides y anestésicos locales en el mismo procedimiento, proponiendo la asociación cuando las inyecciones de esteroides son efectivas pero de corta duración (21). Un aumento del tiempo de exposición a 8 minutos puede asociarse a mejores tasas de éxito y un trabajo comparativo aplicando 4 minutos versus 8 minutos se está desarrollando actualmente en nuestro servicio.

Una debilidad de nuestro trabajo es la ausencia de grupo control. Sin embargo, el valor científico y los aspectos éticos de proponer un grupo control con placebo están discutidos (22). Dado que la punción a nivel del agujero de conjugación puede significar un riesgo, establecer un grupo control donde se simula el procedimiento (grupo Sham) está cuestionado desde el punto de vista ético. Un grupo de estas características fue planteado para realizar en Bélgica por Van Boxem y cols. y su realización fue rechazada por un comité de ética, lo que obligó a los investigadores a realizar un estudio prospectivo de seguimiento (10). Establecer un grupo control con un procedimiento alternativo como tratamiento, como podría ser las inyecciones epidurales de esteroides, presenta cuestionamientos similares, ya que los pacientes seleccionados presentaban fracaso previo a esta alternativa.

Otra crítica que se puede realizar es el número bajo de pacientes que finalmente fueron tratados y los que pudieron ser evaluados a los tres meses. En un estudio realizado por Van Boxem con un diseño similar, un total de 60 pacientes fueron evaluados, el doble que los tratados en el presente estudio (10). El hecho de que solo un tercio de los pacientes tuvieran un efecto clínicamente destacado (mayor a $50 \%$ de mejoría) nos alentó a interrumpir el presente trabajo y a realizar un estudio comparativo y aleatorizado para testear el efecto de utilizar 8 minutos de radiofrecuencia pulsada, utilizando como grupo control el tratamiento estándar durante 4 minutos, estudio actualmente en curso. El seguimiento telefónico en este caso se propuso como mecanismo para reducir la pérdida de controles, en particular a los 3 meses, que se observó en el presente trabajo.

El mecanismo de alivio del dolor en el caso de la radiofrecuencia pulsada es aún objeto de discusión. En esta, la aplicación de calor no produce lesión neural, sino la generación de un campo magnético alrededor de la estructura neural expuesta, produciendo disrupción de las membranas, interfiriendo con la generación de potenciales de acción y de las descargas ectópicas neuronales [8]. Higuchi y cols. mostraron que la aplicación de radiofrecuencia pulsada del ganglio GRD de ratones aumenta la expresión del gen c-Fos a dicho nivel, hallazgo similar al observado por Van Zundert y cols. [23,24]. La relación clínica entre la expresión de c-Fos y el alivio del dolor todavía no ha sido determinada, pero constituye un indicador de que la técnica actúa sobre la transmisión nociceptiva. Otros mecanismos propuestos de la acción de la radiofrecuencia pulsada en modelos de dolor neuropático y radicular incluyen la interferencia con la liberación de sustancias pro-inflamatorias a nivel del conflicto disco-radicular, atenuación del mecanismo de sensibilización central a nivel del asta posterior medular y potenciación de los mecanismos descendentes de analgesia a través de la liberación de noradrenalina y serotonina (25-29). 
Por último, en cuanto a la forma de evaluar los resultados, este es, hasta donde sabemos, el primer reporte utilizando el inventario abreviado de dolor como herramienta para estudiar los efectos analgésicos de la radiofrecuencia pulsada del GRD. Este es un instrumento útil para este fin, que contiene en un único formulario aspectos vinculados con la intensidad del dolor e interferencia de este sobre aspectos importantes de la vida activa y emocional de los pacientes, por lo que lo consideramos una forma completa de evaluación de resultados. En nuestro servicio se utiliza con regularidad y hemos comunicado en una publicación anterior la experiencia en su utilización para evaluar los resultados de las técnicas intervencionistas en el tratamiento de la lumbalgia (12).

\section{CONCLUSIONES}

La aplicación de la radiofrecuencia pulsada sobre el ganglio de la raíz dorsal en un grupo de pacientes con síndrome radicular lumbosacro crónico, refractario a las inyecciones epidurales de esteroides, resultó en una respuesta analgésica satisfactoria en el $40 \%$ de los casos. Estos datos son menores a los encontrados en otros estudios previamente publicados sobre la temática, si bien debe destacarse que estos pacientes eran refractarios tanto a tratamientos intervencionistas como no intervencionistas. Por lo tanto, consideramos que se trata de una técnica válida para el tratamiento del síndrome radicular lumbosacro refractario y pensamos que un aumento del tiempo de exposición al doble puede mejorar las tasas de éxito.

El inventario abreviado de dolor se presentó como una herramienta útil y completa para la evaluación de los resultados. No se observaron complicaciones de la técnica en el grupo de pacientes estudiados.

\section{CONFLICTO DE INTERESES}

Los autores declaran no tener ningún conflicto de intereses.

\section{BIBLIOGRAFÍA}

1. Van Boxem K, Cheng J, Patjin J, van Kleef M, Lataster A, Mekhail N, et al. Lumbosacral Radicular Pain. Pain Pract 2010;10(4):339-58. DOI: 10.1111/j.15332500.2010.00370.x

2. Borenstein D. Low Back Pain. In: Pain Management, Steven D, Waldman MD. Chapter 82. Volume 2. Philadelphia: Ed. Saunders, Elsevier; 2007. p. 749-78.

3. Manchikanti L, Abdi S, Alturi S, et al. An update of comprehensive evidence-based guidelines for interventional techniques in chronic spinal pain. Part II: guidance and recommendations. Pain Physician 2013;16(Suppl. 2):S49-S283.

4. American Society of Anesthesiologists Task Force on Chronic Pain Management; American Society of Regional Anesthesia and Pain Medicine. Practice Guidelines for Chronic Pain Management. An updated report by the American Society of Anesthesiologist Task Force on Chronic Pain Management and the American Society of Regional Anesthesia and Pain Medicine. Anesthesiol 2010;112(4):810-55. DOI: 10.1097/ALN.Ob013e3181c43103.

5. De Palma MJ, Slipman CW. Evidence-informed management of chronic low back pain with epidural steroid injections. Spine J 2008;8(1):45-55. DOI: 10.1016/j.spinee.2007.09.009.

6. Abejón D, García del Valle S, Fuentes ML, Gómez- Arnau $\mathrm{Jl}$, Reig E, van Zundert J. Pulsed radiofrequency in lumbar radicular pain: clinical effects in various etiological groups. Pain Pract 2007;7(1):21-6. DOI: 10.1111/j.15332500.2007.00105.x.

7. Van Boxem K, van Bilsen J, de Meij N, Herrler A, Kessels $A$, Van Zundert J, et al. Pulsed radiofrequency treatment adjacent to the lumbar dorsal root ganglion for the management of lumbosacral radicular syndrome: a clinical audit. Pain Med 2011;12(9):1322-30. DOI: 10.1111/j.15264637.2011.01202.x.

8. Sluijter ME, Cosman E, Rittman I. The effects of pulsed radiofrequency field applied to the dorsal root ganglion-a preliminary report. Pain Clin 1998;11(2):109-17.

9. Van Zundert J, Patijn, Kessels A, Lamé I, Van Suijlekom H, van Kleef M. Pulsed radiofrequency adjacent to the cervical dorsal root ganglion in chronic cervical radicular pain: A double blind sham controlled randomized clinical trial. Pain 2007;127(12):173-82. DOl: 10.1016/j.pain.2006.09.002.

10. Van Boxem K, de Meij N, Kessels A, Van Zundert J, van Kleef M. Pulsed radiofrequency for chronic intractable lumbosacral radicular pain: a six month cohort study. Pain Med 2015;16(6):1155-62. DOl: 10.1111/pme.12670.

11. Keller S, Bann C, Dodd Sh, Schein J, Mendoza T, Cleeland Ch. Validity of the Brief pain Inventory for use in documenting the outcomes of patients with non-cancer pain. Clin J Pain 2004;20(5):309-18.

12. Surbano M, Antunez M, Coutinho I, Machado V, Castroman P. Uso del Brief Pain Inventory (BPI) para la evaluación de las técnicas intervencionistas en el tratamiento de la lumbalgia. Revista El Dolor 2014;(62):10-4.

13. Cleeland CS. Pain assessment: global use of the pain inventory. An Acad Med 1994;23(2):129-38.

14. Gatchel RJ, Mayer TG Choi YH, Chou CR. Validation of a consensus-based minimal clinically important difference (MCID) threshold using an objective functional external anchor. Spine J 2013;13(8):889-93. DOI: 10.1016/j.spinee.2013.02.015.

15. Dworking R, Turk D, Wyrwich K, Beaton D, Cleeland CS, Farrar JT, et al. Interpreting the Clinical Importance of Treatment Outcomes in Chronic Pain Clinical Trials: IMMPACT Recommendations. J Pain 2008;9(2):105-21. DOI: 10.1016/j.jpain.2007.09.005.

16. Trinidad JM, Carnota Al, Failde I, Torres LM. Clinical Study Radiofrequency for the Treatment of Lumbar Radicular Pain: Impact on Surgical Indications. Pain Res Treat 2015;2015:392856. DOl: 10.1155/2015/392856.

17. Samwel H, Salppendel R, Crul BJP, Voerman VF. Psychological predictors of the effectiveness of radiofrequency lesioning of the cervical spinal dorsal ganglion (RF-DRG). Eur J Pain 2000;4(2):149-55. DOI: 10.1053/eujp.2000.0165.

18. Van Boxem K, de Meij N, Patijn J, Wilmink J, van Kleef M, Van Zundert J, et al. Predictive factors for successful outcome of pulsed radiofrequency treatment in patients with intractable lumbosacral radicular pain. Pain Med 2016;17(7):1233-40. DOI: 10.1093/pm/pnv052.

19. Lee CC, Chen CJ, Chou CC, Wang HY, Chung WY, Peng GS, et al. Lumbar Dorsal Root Ganglion as a prognostic tool before pulsed radiofrequency: a randomized, prospective, and 
comparative study on cost-effectiveness. World Neurosurg 2018;112:e157-e164. DOI: 10.1016/j.wneu.2017.12.183.

20. Kim SJ, Park SJ, Yoon KB, Kim SH. Predictors of the analgesic efficacy of pulsed radiofrequency treatment in patients with chronic lumbosacral radicular pain: a retrospective observational study. J Pain Res 2018;11:1223-30. DOI: 10.2147/JPR.S164414.

21. Koh W, Choi SS, Karm MH, Suh JH, Leem JG, Lee JD, et al. Treatment of chronic lumbosacral radicular pain using adjuvant pulsed radiofrequency: a randomized controlled study. Pain Med 2015;16(3):432-41. DOI: 10.1111/pme.12624.

22. Manchikanti L, Cash KA, Pampati V, Falco FJE. Transforaminal epidural injections in chronic lumbar disc herniation: a randomized, double-blind, active-control trial. Pain Physician 2014;17(4):489-501.

23. Higuchi Y, Nashold BS Jr, Sluijter M, Cosman E, Pearlstein $\mathrm{RD}$. Exposure of the dorsal root ganglion in rats to pulsed radiofrequency currents activates dorsal horn lamina I and II neurons. Neurosurgery 2002;50(4):850-5.

24. Van Zundert J, de Louw AJ, Joosten EA, Kessels AG, Honig W, Dederen PJ, et al. Pulsed and continuous radiofrequency current adjacent to the cervical dorsal root ganglion of the rat induces late cellular activity in the dorsal horn. Anesthesiology 2005;102(1):125-31.
25. Park HW, Ahn SH, Kim SJ, Seo JM, Cho YW, Jang SH, et al. Changes in spinal cord expression of fractalkine and its receptor in a rat model of disc herniation by autologous nucleus pulposus. Spine 2011;36(12):753-60.

26. Kim SJ, Kim WR, Kim HS, Park HW, Cho YW, Jang $\mathrm{SH}$, et al. Abnormal spontaneous activities on needle electromyography and their relation with pain behavior and nerve fiber pathology in a rat model of lumbar disc herniation. Spine 2011;36(24):1562-7. DOI: 10.1097/ BRS.Ob013e318210aa10.

27. Erdine S, Bilir A, Cosman ER, Cosman ER Jr. Ultrastructural changes in axons following exposure to pulsed radiofrequency fields. Pain Practice 2009;9(6):407-17. DOI: 10.1111/j.1533-2500.2009.00317.x.

28. Hamann W, Abou-Sherif S, Thompson S, Hall S. Pulsed radiofrequency applied to dorsal root ganglia causes a selective increase in ATF3 in small neurons. Eur J Pain 2006;10(2):171-6. DOI: 10.1016/j. ejpain.2005.03.001.

29. Hagiwara S, Iwasaka H, Takeshima N, Noguchi T. Mechanisms of analgesic action of pulsed radiofrequency on adjuvant-induced pain in the rat: Roles of descending adrenergic and serotonergic systems. Eur J Pain 2009;13(3):24952. DOI: 10.1016/j.ejpain.2008.04.013. 